\title{
Myeloid cell leukemia-1 is a molecular indicator for malignant transformation of oral lichen planus
}

\author{
JI-AE SHIN $^{1 *}$, JAE-MIN SEO ${ }^{2 *}$, SEJUN OH ${ }^{1}$, SUNG-DAE CHO $^{1}$ and KYUNG-EUN LEE ${ }^{3,4}$ \\ ${ }^{1}$ Department of Oral Pathology, School of Dentistry and Institute of Biodegradable Material, Institute of Oral Bioscience, \\ Brain Korea 21 Project; ${ }^{2}$ Department of Prosthodontics, School of Dentistry and Institute of Oral Bioscience, \\ Biomedical Research Institute; ${ }^{3}$ Department of Oral Medicine, School of Dentistry, Institute of Oral Bioscience, \\ Chonbuk National University, Jeonju 561-756; ${ }^{4}$ Research Institute of Clinical Medicine of Chonbuk National University \\ - Biomedical Research Institute of Chonbuk National University Hospital, Jeonju 561-712, Republic of Korea
}

Received December 15, 2014; Accepted December 14, 2015

DOI: $10.3892 / \mathrm{ol} .2016 .4083$

\begin{abstract}
Oral lichen planus (OLP), characterized by a chronic mucocutaneous inflammatory condition, is a common disease of the oral cavity. Retrospective and prospective epidemiological data suggest that OLP is considered to have malignant potential. However, it is unclear as to which types of molecules may cause malignant transformation of OLP. In the present study, the presence of myeloid cell leukemia-1 (Mcl-1) and B-cell lymphoma-2 (Bcl-2) was studied by western blot analysis in 11 OLP and three normal oral mucosa (NOM) samples and in two human oral cancer cell lines. The functional role of Mcl-1 in oral cancer cells was analyzed using a trypan blue exclusion assay and soft agar assay. Mcl-1 was strongly expressed in the OLP and the two oral cancer cell lines compared with NOM, whereas Bcl-2 was not. Sorafenib and mithramycin A decreased cell viability in MC-3 and HSC-3 oral cancer cells and at same concentration they reduced the expression level of Mcl-1 in the two cell lines. The two chemicals affected Mcl-1 protein and significantly inhibited neoplastic cell transformation in the two cell lines. We suggest that the malignant potential of OLP may be associated with the expression of Mcl-1, and that downregulation of Mcl-1 may prevent malignant transformation of OLP to oral cancer.
\end{abstract}

Correspondence to: Professor Sung-Dae Cho, Department of Oral Pathology, School of Dentistry and Institute of Biodegradable Material, Institute of Oral Bioscience, Brain Korea 21 Project, Chonbuk National University, 567 Baejedaero, Jeonju 561-756, Republic of Korea

E-mail: efiwdsc@jbnu.ac.kr

Professor Kyung-Eun Lee, Department of Oral Medicine, School of Dentistry, Institute of Oral Bioscience, Chonbuk National University, 567 Baejedaero, Jeonju 561-756, Republic of Korea

E-mail:1ke@chonbuk.ac.kr

${ }^{*}$ Contributed equally

Key words: oral lichen planus, malignant transformation, myeloid cell leukemia-1, oral cancer, B-cell lymphoma-2

\section{Introduction}

Oral lichen planus (OLP), characterized by a chronic mucocutaneous inflammatory condition (1), is considered to be a T-cell-mediated immunological process, although the etiology and pathogenesis of OLP is not completely understood (2). The prevalence of OLP has been estimated to be between 0.5 and $3 \%$ in a number of studies (3-5). A previous review reported an overall age-standardized prevalence of $1.27 \%(0.96 \%$ in males and $1.57 \%$ in females) (6). These data reflect that OLP is a common mucosal disease of the oral cavity.

One of the most significant issues concerning OLP is the question of its potential for malignant transformation into oral squamous cell carcinoma (OSCC). Since the World Health Organization (WHO) developed diagnostic criteria for OLP that included clinical and histopathological standards in 1978 (7), certain authors have considered OLP to be a precancerous lesion based on retrospective and prospective epidemiological data (8-11). Van der Meij et al proposed a modification of the WHO diagnostic criteria for OLP to include the definition of an entity referred to as 'oral lichenoid lesion' (OLL) and to differentiate between OLL and OLP clinically and histopathologically. By applying clinically and histologically diagnostic modified WHO criteria, these authors reported that patients with OLL have an increased risk of oral cancer, but this increased risk was not detected in patients with OLP (12).

A cytokine-based microenvironment arising from the chronic inflammation of OLP may induce genetic alterations of epithelial cells to progress to malignancy (13). Although a permanent cure of OLP is not yet possible, various treatment regimens have been designed to improve management of the symptoms of OLP (14). Several studies have suggested that the expression of apoptosis- and cell cycle-regulating proteins, including p53 protein, B-cell lymphoma-2 (Bcl-2) and Bax, was also involved in the transformation process $(15,16)$. However, the mechanisms causing the malignant transformation of OLP remain unclear.

Therefore, the purpose of the present study was to detect and compare several proteins including myeloid cell 
A

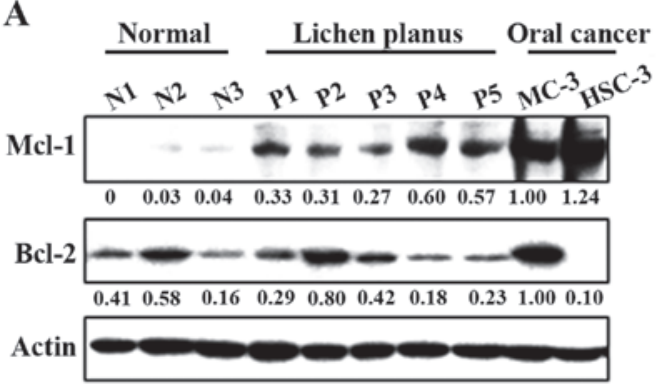

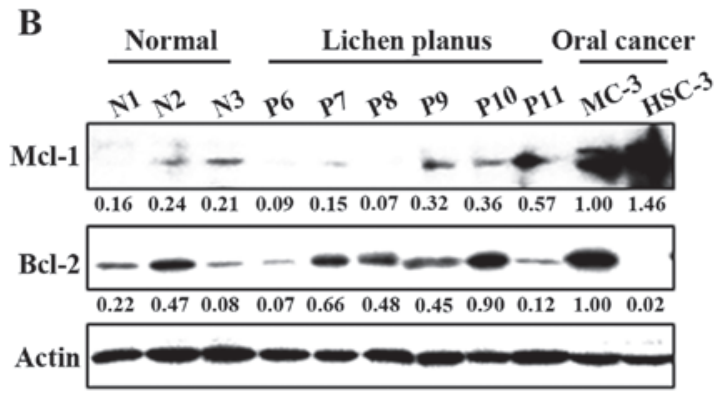

Figure 1. Myeloid cell leukemia-1 (Mcl-1) expression is associated with the pathogenesis of oral lichen planus (OLP). (A and B) The difference in Mcl-1 expression between normal oral mucosa and OLP. Whole-cell lysates were analyzed by western blot analysis using antibodies against Mcl-1 and B-cell lymphoma-2 (Bcl-2). Actin was used as a loading control.

leukemia-1 (Mcl-1) as possible molecules involved in the malignant transformation of OLP in normal oral mucosa (NOM), OLP and two human oral cancer cell lines (MC-3 and HSC-3). We also suggest a strategy on how to prevent malignant transformation of OLP.

\section{Materials and methods}

Patients and tissue samples. A total of 14 outpatients were investigated by two dentists between 2012 and 2013. Three normal samples of gingiva without pathological lesions were provided from outpatients (one male and two females; age range from 49 to 56 years) for implant surgery at the Department of Prosthodontics, Chonbuk National University Hospital (Jeonju, Korea). Eleven samples of outpatients (three males and nine females; age range from 49 to 73 years) with OLP were obtained from the Department of Oral Medicine, of the same hospital. The materials and methods in the present study were approved by the ethics committee of Chonbuk National University Hospital (CUH2013-04-031-001) and written informed consent was obtained from all patients.

Cell lines and cell culture. MC-3 cells (human mucoepidermoid carcinoma) were provided by Professor Junzheng $\mathrm{Wu}$ (Fourth Military Medical University, Xi'an, China) and HSC-3 cells (human oral squamous carcinoma) were obtained from Professor Shindo (Hokkaido University, Hokkaido, Japan). The two cell lines were cultured in Dulbecco's modified Eagle's medium/F12 supplemented with $10 \%$ fetal bovine serum (FBS; Welgene, Daegu, Korea) and antibiotics at $37^{\circ} \mathrm{C}$ in a $5 \% \mathrm{CO}_{2}$ incubator.

Reagents and antibodies. A DC protein assay kit was obtained from Bio-Rad Laboratories, Inc. (Madison, WI, USA). Trypan blue solution was purchased from Gibco Life Technologies (Paisley, UK). Antibodies against Mcl-1 and Bcl-2 were obtained from Cell Signaling Technology, Inc. (Charlottesville, VA, USA). Actin was purchased from Santa Cruz Biotechnology, Inc. (Santa Cruz, CA, USA).

Western blot analysis. To determine the levels of protein expression, whole-cell lysates were prepared with lysis buffer and protein concentrations were measured. Equal amounts of proteins were loaded on sodium dodecyl sulphate-polyacrylamide gel, transferred to polyvinylidene membranes and assessed using an enhanced chemiluminescence western blotting reagent (Luminol; Santa Cruz Biotechnology, Inc.).

Trypan blue exclusion assay. The effects of sorafenib (LC Laboratories, Woburn, MA, USA) and mithramycin A (Sigma-Aldrich, St. Louis, MO, USA) on cell viability were determined using a trypan blue exclusion assay. Cells were incubated with sorafenib and mithramycin A for $48 \mathrm{~h}$, stained with trypan blue $(0.4 \%)$, and then viable cells were counted using a hemocytometer (Thermo Fisher Scientific, Waltham, MA, USA).

Anchorage-independent growth assay. MC-3 and HSC-3 cells were treated with various concentrations of sorafenib or mithramycin A in $1 \mathrm{ml} 0.3 \%$ basal medium Eagle's agar containing $10 \%$ FBS. The culture was incubated at $37^{\circ} \mathrm{C}$ in a $5 \% \mathrm{CO}_{2}$ incubator for 20 days, and then colonies were counted.

Statistical analysis. Student's t-test was used to determine the significance of differences between the control and treatment groups; $\mathrm{P}<0.05$ was considered to indicate a statistically significant difference.

\section{Results}

Correlation between Mcl-1 expression and pathogenesis of OLP. To clarify the potential role of anti-apoptotic Bcl-2 family proteins on the pathogenesis of OLP, we compared three samples of NOM and eleven samples from outpatients with proven OLP. Notably, the expression of Mcl-1 was significantly higher in the OLP than in the NOM samples, although in both samples it was lower than in the MC-3 and HSC-3 human oral cancer cell lines (Fig. 1A and B). However, there was no association between $\mathrm{Bcl}-2$ expression and the pathogenesis of OLP. These results indicate that Mcl-1 expression may contribute to malignant transformation in OLP.

Effects of sorafenib and mithramycin A on cell growth and Mcl-1 expression in human oral cancer cell lines. To investigate the potential effects of sorafenib and mithramycin A on cell viability in human oral cancer cell lines, we treated the cells with dimethyl sulfoxide or various concentrations of sorafenib and mithramycin A for 48 h. As shown in Fig. 2A and $\mathrm{B}$, the two chemicals notably decreased cell viability in MC-3 and HSC-3 cell lines. To evaluate whether the effects 

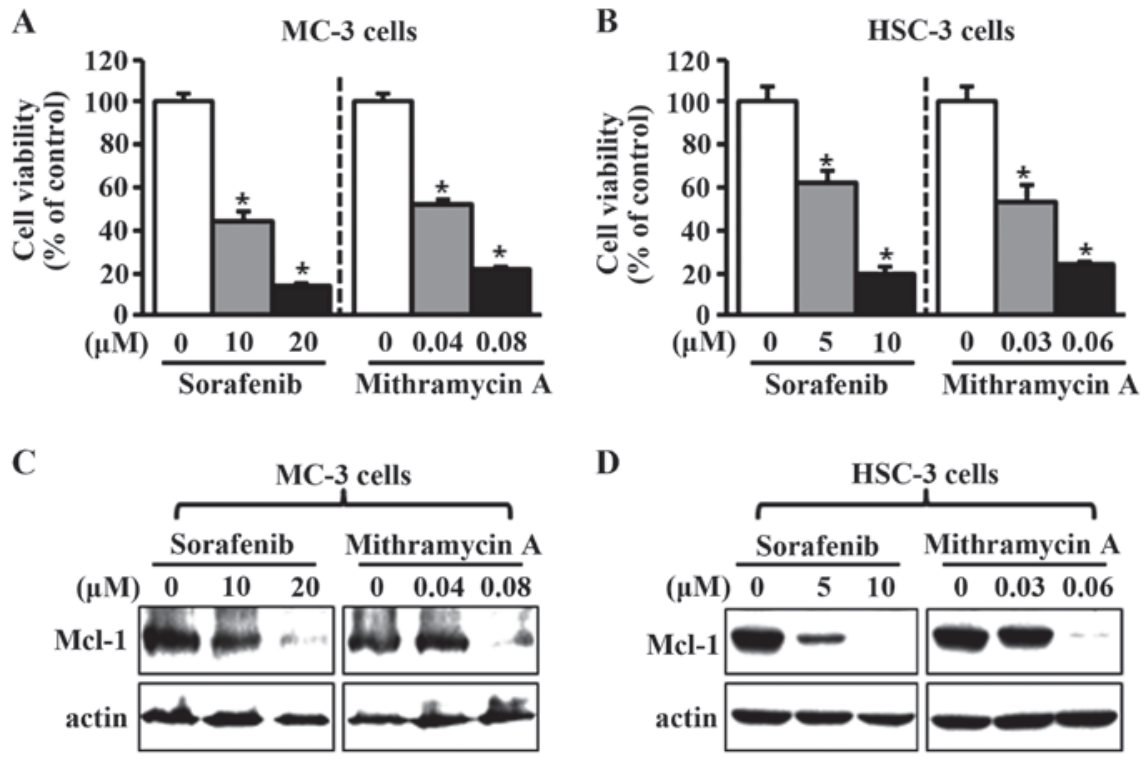

Figure 2. Sorafenib and mithramycin A decrease cell viability and Mcl-1 expression in human oral cancer cell lines. (A and B) MC-3 and HSC-3 cells were treated with dimethyl sulfoxide (DMSO) or various concentrations of sorafenib and mithramycin A for $48 \mathrm{~h}$. The effects on cell viability were determined using a trypan blue exclusion assay. The results are expressed the means \pm standard deviation of three experiments. ${ }^{*} \mathrm{P}<0.05$ compared with the DMSO-treated group. (C and D) Whole-cell lysates were analyzed by western blot analysis using an antibody against Mcl-1. Actin was used as a loading control.

A
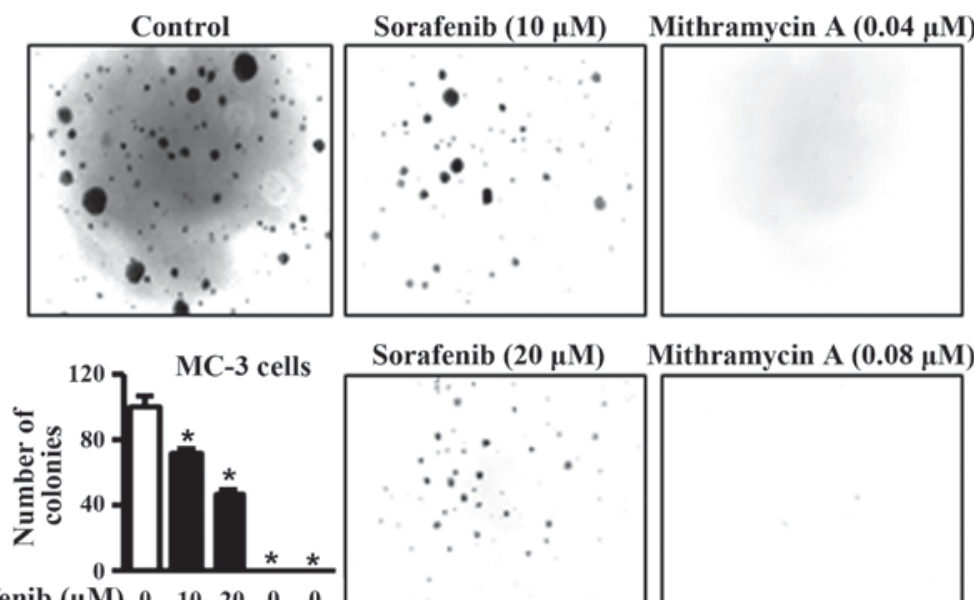

Sorafenib $(20 \mu \mathrm{M}) \quad$ Mithramycin $\mathrm{A}(\mathbf{0 . 0 8} \mu \mathrm{M})$

Sorafenib $(\mu \mathrm{M}) \quad 0 \quad 10 \quad 20 \quad 0 \quad 0$

Mithramycin A $(\mu \mathrm{M}) \quad 0 \quad 0 \quad 00.040 .08$

B
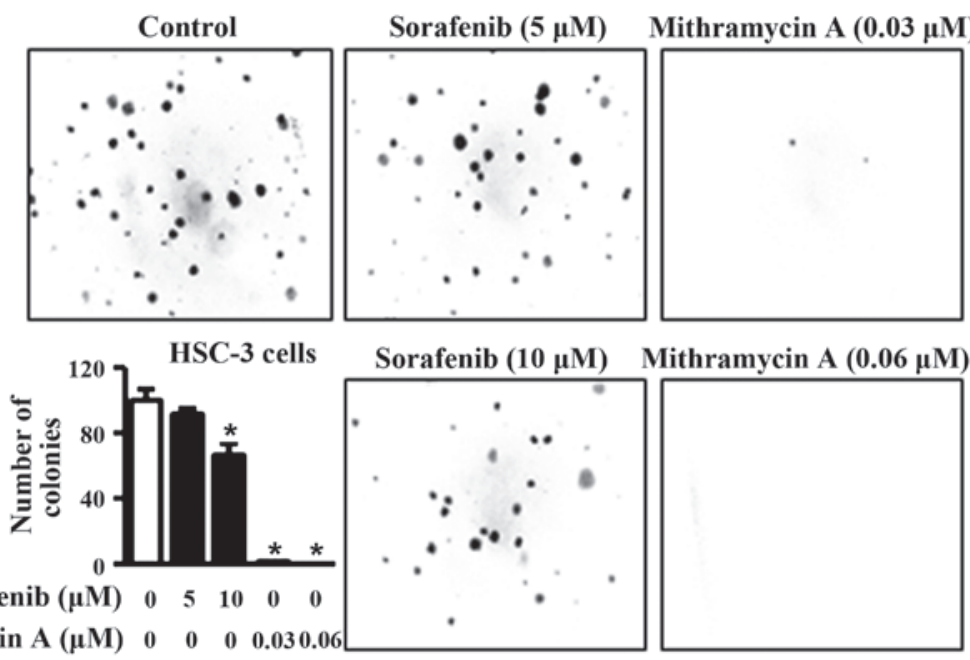

Figure 3. Sorafenib and mithramycin A decrease anchorage-independent growth in human oral cancer cell lines. (A and B) For the soft agar assay, MC-3 and HSC-3 cells were treated with sorafenib and mithramycin A for 20 days, and then colonies were counted. The results are expressed as the means \pm standard deviation of three experiments. " $\mathrm{P}<0.05$ compared with the dimethyl sulfoxide-treated group. 
of sorafenib and mithramycin A on growth inhibition were associated with the regulation of Mcl-1 expression, we used western blot analysis. As shown in Fig. 2C and D, sorafenib and mithramycin A reduced Mcl-1 expression in the two cell lines. To further confirm the anti-proliferative effects of sorafenib and mithramycin $\mathrm{A}$, we carried out anchorage-independent colony formation assay. The results in Fig. 3A and B reveal that sorafenib and mithramycin A effectively decreased neoplastic cell transformation in MC-3 and HSC-3 cells. These results indicate that sorafenib and mithramycin A may have the ability to prevent neoplastic transformation from OLP to oral cancer through downregulation of Mcl-1.

\section{Discussion}

Apoptosis, also termed programmed cell death, may be triggered by various stimuli from outside or inside the cell and characterized by well-defined biochemical and morphological changes (17). Bcl-2 family members control mitochondrial outer membrane permeabilization, and may be either pro-apoptotic (Bax, Bak, Bid, Bim and Bad, among others) or anti-apoptotic (Bcl-2, Bcl-xL and Mcl-1, among others) (18). Bcl-2 has the function of inhibiting apoptosis at various stages and is strongly associated with cancer development, increasing the genetically altered cell survival rate. In oral cancer, it is observed from the initial stages of carcinogenesis up to the appearance of metastasis (19-21). Mcl-1 has been reported to be a critical survival-promoting molecule in hematopoietic cells, and its overexpression is known to be associated with a variety of human hematopoietic and lymphoid cancers (22). The anti-apoptotic splice variant of Mcl-1 was also augmented in human oral cancer (23), and our previous study demonstrated that the overexpression of Mcl-1 may be strongly correlated with oral cancer development (24).

In the present study, we hypothesized that Bcl-2 or Mcl-1 may play a significant role in potential malignant transformation of OLP. To test this hypothesis, our approach was to evaluate their expression levels in OLP samples compared with NOM samples and MC-3 and HSC-3 human oral cancer cell lines. Our results demonstrated that Mcl-1 expression was notably higher in patient samples of OLP than NOM, with both levels being lower than in MC-3 and HSC-3 human oral cancer cell lines, while there was no difference in Bcl-2 expression levels among the samples and cell lines. We demonstrated the difference in expression levels of Mcl-1 protein among NOM, OLP and human oral cancer cell lines for the first time. These results indicate that Mcl-1 expression may contribute to the pathogenesis of OLP and its possible progression to oral cancer. In a previous comparative study (25), no statistically significant differences between the expression of Bcl-2 in OLP and OSCC were observed, which is consistent with our results. This suggests no association between Bcl-2 expression and pathogenesis of OLP and progression to oral malignancy.

Several studies have reported a high expression level of Mcl-1 in tumor cells of human primary squamous cell carcinoma (SCC) and SCC cell lines $(24,26)$. It has been demonstrated that the blockage of its function may play a potentially critical and novel role by the use of either specific
siRNA or inhibitor in the treatment of SCC (26). To date, several therapeutic strategies have been designed to abrogate the anti-apoptotic function of Mcl-1 in a variety of tumor types $(27,28)$. Therefore, we hypothesized that downregulation of Mcl-1 may prevent malignant transformation of OLP to oral cancer. Previously, in vitro and in vivo studies by our group reported that sorafenib and mithramycin A regulated Mcl-1 protein to inhibit the proliferation of oral cancer $(24,29)$. Thus, we assessed the anti-proliferative effect of sorafenib and mithramycin A on MC-3 and HSC-3 cells in order to demonstrate the function of Mcl-1 in the malignant transformation of OLP to oral cancer. The results revealed that the two chemicals notably decreased cell viability and neoplastic cell transformation in the two cell lines. Although we did not directly treat the OLP specimens with sorafenib and mithramycin A, these results indicate that the inhibition of Mcl-1 may prevent malignant transformation of OLP to oral cancer.

In summary, we demonstrated that Mcl-1 protein is expressed at a higher level in OLP than in NOM, and lower than in oral cancer cell lines. We also observed that the decreases in Mcl-1 protein by sorafenib and mithramycin A reduced cell growth and inhibited neoplastic cell transformation. Our study indicates that the malignant potential of OLP may be correlated with the expression of Mcl-1, and a strategy to downregulate Mcl-1 may prevent its malignant potential. However, further studies are required in order to support this hypothesis.

\section{Acknowledgements}

This study was supported by the Fund of Biomedical Research Institute, Chonbuk National University Hospital and Basic Science Research Program through the National Research Foundation of Korea (NRF) funded by the Ministry of Education, Science and Technology (2014R14A1005309).

\section{References}

1. Barnes L, Eveson JW, Reichart P and Sidransky D (eds): Tumor of the oral cavity and oropharynx. In: Pathology and Genetics of Head and Neck Tumours (World Health Organization Classification of Tumours). IARC Press, Lyon, 2005.

2. Roopashree MR, Gondhalekar RV, Shashikanth MC, George J, Thippeswamy SH and Shukla A: Pathogenesis of oral lichen planus - a review. J Oral Pathol Med 39: 729-734, 2010.

3. Farhi D and Dupin N: Pathophysiology, etiologic factors and clinical management of oral lichen planus, part I: facts and controversies. Clin Dermatol 28: 100-108, 2010.

4. Ismail SB, Kumar SK and Zain RB: Oral lichen planus and lichenoid reactions: etiopathogenesis, diagnosis, management and malignant transformation. J Oral Sci 49: 89-106, 2007.

5. Parashar P: Oral lichen planus. Otolaryngol Clin North Am 44: 89-107, 2011.

6. McCartan BE and Healy CM: The reported prevalence of oral lichen planus: a review and critique. J Oral Pathol Med 37: 447-453, 2008.

7. Kramer IR, Lucas RB, Pindborg JJ and Sobin LH: Definition of leukoplakia and related lesions: an aid to studies on oral precancer. Oral Surg Oral Med Oral Pathol 46: 518-539, 1978.

8. Silverman S Jr, Gorsky M and Lozada-Nur F: A prospective follow-up study of 570 patients with oral lichen planus: persistence, remission and malignant association. Oral Surg Oral Med Oral Pathol 60: 30-34, 1985.

9. Murti PR, Daftary DK, Bhonsle RB, Gupta PC, Mehta FS and Pindborg JJ: Malignant potential of oral lichen planus: observations in 722 patients from India. J Oral Pathol 15: 71-77, 1986. 
10. Silverman S Jr, Gorsky M, Lozada-Nur F and Giannotti K: A prospective study of findings and management in 214 patients with oral lichen planus. Oral Surg Oral Med Oral Pathol 72: 665-670, 1991.

11. Sigurgeirsson B and Lindelöf B: Lichen planus and malignancy. An epidemiologic study of 2071 patients and a review of the literature. Arch Dermatol 127: 1684-1688, 1991.

12. van der Meij EH, Mast H and van der Waal I: The possible premalignant character of oral lichen planus and oral lichenoid lesions: a prospective five-year follow-up study of 192 patients Oral Oncol 43: 742-748, 2007.

13. Mignogna MD, Fedele S, Lo Russo L, Lo Muzio L and Bucci E: Immune activation and chronic inflammation as the cause of malignancy in oral lichen planus: is there any evidence? Oral Oncol 40: 120-130, 2004.

14. Lodi G, Scully C, Carrozzo M, Griffiths M, Sugerman PB and Thongprasom K: Current controversies in oral lichen planus: report of an international consensus meeting. Part 2. Clinical management and malignant transformation. Oral_Surg Oral Med Oral Pathol Oral Radiol Endod 100: 164-178, 2005.

15. Sousa FA, Paradella TC, Carvalho YR and Rosa LE: Immunohistochemical expression of PCNA, p53, bax and bcl-2 in oral lichen planus and epithelial dysplasia. J Oral Sci 51: 117-121, 2009.

16. de Sousa FA, Paradella TC, Carvalho YR and Rosa LE Comparative analysis of the expression of proliferating cell nuclear antigen, p53, bax and bcl-2 in oral lichen planus and oral squamous cell carcinoma. Ann Diagn Pathol 13: 308-312, 2009.

17. Nagata S: Apoptosis by death factor. Cell 88: 355-365, 1997.

18. Kuwana T, Bouchier-Hayes L, Chipuk JE, Bonzon C, Sullivan BA, Green DR and Newmeyer DD: BH3 domains of BH3-only proteins differentially regulate Bax-mediated mitochondria membrane permeabilization both directly and indirectly. Mol Cell 17: 525-535, 2005.

19. Jordan RC, Catzavelos GC, Barrett AW and Speight PM: Differential expression of bcl-2 and bax in squamous cell carcinomas of the oral cavity. Eur J Cancer B Oral Oncol 32B: 394-400, 1996.
20. Singh BB, Chandler FW Jr, Whitaker SB and Forbes-Nelson AE: Immunohistochemical evaluation of bcl-2 oncoprotein in oral dysplasia and carcinoma. Oral Surg Oral Med Oral Pathol Oral Radiol Endod 85: 692-698, 1998.

21. Sulkowska M, Famulski W, Chyczewski L and Sulkowski S Evaluation of p53 and bcl-2 oncoprotein expression in precancerous lesions of the oral cavity. Neoplasma 48: 94-98, 2001.

22. Warr MR and Shore GC: Unique biology of Mcl-1: therapeutic opportunities in cancer. Curr Mol Med 8: 138-147, 2008.

23. Mallick S, Patil R, Gyanchandani R, Pawar S, Palve V, Kannan S, Pathak KA, Choudhary M and Teni TR: Human oral cancers have altered expression of Bcl-2 family members and increased expression of the anti-apoptotic splice variant of Mcl-1. J Pathol 217: 398-407, 2009.

24. Shin JA, Jung JY, Ryu MH, Safe S and Cho SD: Mithramycin A inhibits myeloid cell leukemia-1 to induce apoptosis in oral squamous cell carcinomas and tumor xenograft through activation of Bax and oligomerization. Mol Pharmacol 83: 33-41, 2013

25. Leyva-Huerta ER, Ledesma-Montes C, Rojo-Botello RE and Vega-Memije E: P53 and bcl-2 immunoexpression in patients with oral lichen planus and oral squamous cell carcinoma. Med Oral Patol Oral Cir Bucal 17: e745-e750, 2012.

26. Nagata M, Wada K, Nakajima A, Nakajima N, Kusayama M, Masuda T, Iida S, Okura M, Kogo M and Kamisaki Y: Role of myeloid cell leukemia-1 in cell growth of squamous cell carcinoma. J Pharmacol Sci 110: 344-353, 2009.

27. Huang S, Okumura K and Sinicrope FA: BH3 mimetic obatoclax enhances TRAIL-mediated apoptosis in human pancreatic cancer cells. Clin Cancer Res 15: 150-159, 2009.

28. Rahmani M, Davis EM, Bauer C, Dent P and Grant S: Apoptosis induced by the kinase inhibitor BAY 43-9006 in human leukemia cells involves down-regulation of Mcl-1 through inhibition of translation. J Biol Chem 280: 35217-35227, 2005.

29. Yu HJ, Shin JA, Jung JY, Nam JS, Hong IS, Cho NP and Cho SD: Inhibition of myeloid cell leukemia-1: Association with sorafenib-induced apoptosis in human mucoepidermoid carcinoma cells and tumor xenograft. Head Neck 37: 1326-1335, 2015. 